\title{
Dynamics of the lower thermosphere over South Pole from meteor radar wind measurements
}

\author{
J. M. Forbes ${ }^{1}$, Yu. I. Portnyagin ${ }^{2}$, N. A. Makarov², S. E. Palo ${ }^{1}$, E. G. Merzlyakov ${ }^{2}$ and X. Zhang ${ }^{1}$ \\ ${ }^{1}$ Department of Aerospace Engineering Sciences, Campus Box 429, University of Colorado, Boulder, CO 80309-0429, U.S.A. \\ ${ }^{2}$ Institute for Experimental Meteorology, Obninsk, Russia
}

(Received August 17, 1998; Revised March 15, 1999; Accepted June 16, 1999)

\begin{abstract}
A meteor radar was operated at Amundsen-Scott Station, South Pole, from January 19, 1995 through January 26, 1996 and from November 21, 1996 through January 27, 1997. Hourly wind measurements were obtained nearly continuously over these time periods, at an approximate altitude of $95 \mathrm{~km}$ and at about $2^{\circ}$ latitude from South Pole along the longitude meridians $0^{\circ}, 90^{\circ} \mathrm{E}, 90^{\circ} \mathrm{W}$, and $180^{\circ}$. The scientific advances achieved to date through analyses of these data are presented, including updates to several of our previously published works. The findings addressed herein include the following: (1) Strong divergences of zonal-mean meridional winds occasionally occur over South Pole, implying extreme vertical winds; (2) The monthly mean zonally asymmetric (zonal wavenumber $s=1$ ) wind component varies during the year in a manner consistent with migration of the center of the polar vortex with respect to the geographic (rotational) pole; (3) Strong $(>15 \mathrm{~m} / \mathrm{s})$ westward-propagating migrating diurnal $(s=1)$ and non-migrating semidiurnal $(s=1)$ oscillations exist except during winter months; (4) Long-period ( $\sim 2-10$ days) waves exist during winter months which are primarily eastward-propagating; (5) Intradiurnal (periods $\sim 6-11.5$ hours) westward-propagating oscillations exist, which are thought to be gravitational normal modes, or "Lamb" waves.
\end{abstract}

\section{Introduction}

On January 19, 1995, through collaborative efforts between the University of Colorado (Boulder, U.S.A.) and the Institute for Experimental Meteorology (Obninsk, Russia), a meteor radar capable of continuously measuring winds near $95 \mathrm{~km}$ in four azimuthal directions (longitudinal meridians $0^{\circ}, 90^{\circ} \mathrm{E}, 180^{\circ}$, and $90^{\circ} \mathrm{W}$ ) began operations at AmundsenScott Station, South Pole. The radar operated continuously until January 26, 1996, thus providing horizontal wind measurements covering all seasons of the year. A second campaign was conducted during the summer season from November 21, 1996, through January 27, 1997.

The dynamics of the neutral upper mesosphere/lower thermosphere (MLT) region over the South Pole has previously been investigated using optical-based experimental methods (Collins et al., 1992; Hernandez et al., 1992a, 1992b, 1993, 1995; Fraser et al., 1993; Sivjee and Walterscheid, 1994). These studies have revealed some basic characteristics of atmospheric motions close to the rotational poles; for example, the flow field possesses a zonal wavenumber one $(s=1)$ structure, while scalar variables (such as temperature and $\mathrm{OH}$-emission rates) are characterized by $s=0$ (i.e., zonal symmetry). The above measurements have also noted the wintertime presence of westward-propagating oscillations with periods near 10 and 12 hours, and 2-4 day eastward-propagating oscillations. However, the aforementioned optical techniques are limited in their ability to carry out long-term continuous measurements (clear sky condi-

Copy right $($ C The Society of Geomagnetism and Earth, Planetary and Space Sciences (SGEPSS); The Seismological Society of Japan; The Volcanological Society of Japan; The Geodetic Society of Japan; The Japanese Society for Planetary Sciences. tions in the absence of significant sun and moon illumination of the sampling volumes). As a result, little information exists on the persistence or variability characteristics of the above oscillations. In addition, no wind measurements whatsoever have been made in the MLT region over the South Pole during non-winter months prior to the present series of observations.

In Section 2 we describe the meteor radar system and its operational capabilities. The measurement results and their interpretations are discussed in Sections 3 and 4. Conclusions are made in the final part of the paper.

\section{The Radar System}

The meteor radar system and various error estimates are described by Portnyagin et al. $(1997,1998)$ and Palo et al. (1998). An abbreviated description is provided here.

In the meteor radar technique, winds are detected by measuring the Doppler shift of coherent radio reflections from the ionized trails produced by meteor ablation in the upper atmosphere. The meteor trails are several kilometers in length, with an initial radius of about 1 meter. The South Pole system operates at a frequency of $33.57 \mathrm{Mhz}$, with a peak power of $7 \mathrm{~kW}$, a pulse width of 120 microseconds and a pulse repetition frequency of either 100 or $300 \mathrm{~Hz}$ (see below). The radar operates in a monostatic configuration with four five-element Yagi transmitting/receiving antennae, directed in the horizontal plane along the $0^{\circ}, 90^{\circ} \mathrm{E}, 180^{\circ}$, and $90^{\circ} \mathrm{W}$ geographical meridians. In the vertical plane the antennae are directed at an elevation angle of $28^{\circ}$. The two-way halfpower-halfwidth of the antenna beams are about $20^{\circ}$ in both azimuth and elevation, which translate to minor uncer- 
tainties in our wind estimates (Palo et al., 1998). The hourly mean winds, which are assumed to be horizontal, represent Gaussian-weighted averages centered near $95 \mathrm{~km}$ above sea level. As a result, the system is only sensitive to wave structures with vertical wavelengths exceeding about $20 \mathrm{~km}$ (Palo et al., 1998). (N.B.: The only wind component measured from South Pole is the meridional wind, taken here to be positive in the southward direction).

The system operates in two modes. In the search mode, the atmosphere is "interrogated" with frequency $100 \mathrm{~Hz}$ for the appearance of reflections from meteor trails. When a meteor echo is detected in any direction, the search mode is stopped and pulses with repetition frequency of $300 \mathrm{~Hz}$ are radiated. A number of measures exist to ensure that "good" echoes, i.e., those that lead to reliable Doppler shifts (wind measurements), are selected while others are rejected. In particular, the interrogation/acquisition sequence automatically eliminates a number of spurious and unreliable echoes from the analysis. Auroral echoes have a number of characteristic features which allow them to be identified and then rejected. About 5000 valid meteor echoes are acquired per day.

\section{Measurement Results-The "Mean" Compo- nent of the Wind}

As illustrated in Fig. 1, the meridional wind field in the vicinity of South Pole can be separated into a zonal mean (or zonally symmetric) component, and the deviation from the zonal mean (or the zonally asymmetric component). For the former, a positive (negative) value implies net convergence (divergence) towards (away from) the pole. The zonally asymmetric component can be viewed as the vector of the wind blowing over the South Pole. For the latter, the winds corresponding to meridians $180^{\circ}$ apart are anticorrelated.

Portnyagin et al. (1997) analyzed the day-to-day variability of the daily- and zonal-mean meridional wind, and the month-to-month variation of the monthly mean zonally asymmetric component of the wind. The daily zonal mean

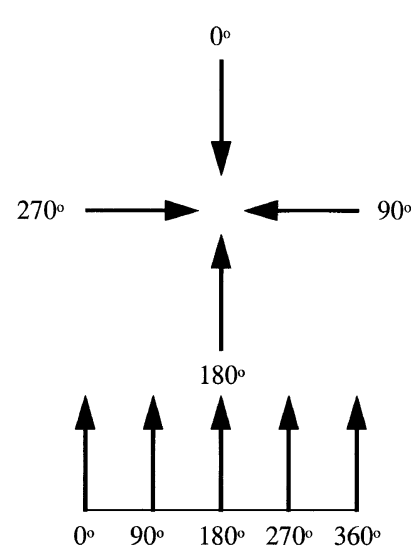

zonally symmetric component
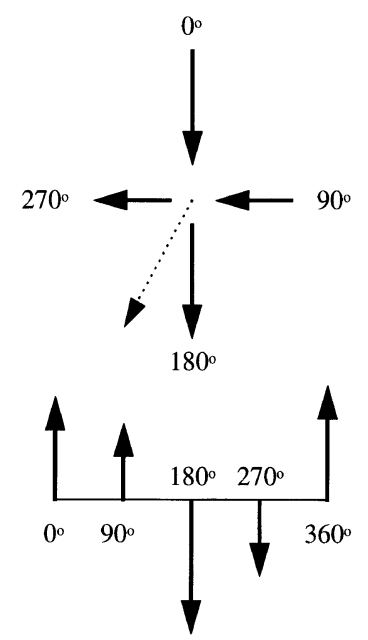

zonally asymmetric component
Fig. 1. Schematic of the zonally symmetric and zonally asymmetric components of the meridional wind over the pole. meridional winds for the first year of radar operations are plotted in Fig. 2. Maximum errors for each data point are $8 \mathrm{~m} \mathrm{~s}^{-1}$ (Portnyagin et al., 1997). As expected, the mean meridional wind is generally small; however, there are two periods, one in April and one in November, when the mean winds appear particularly large. The April event is examined more closely in Fig. 3. Also included in this Figure are values of the daily planetary magnetic index, Ap. It is evident that the "wind divergence event" began to develop in late March, reached a maximum by April 10, and subsided precipitously over the next few days. The fact that the event began during extremely quiet magnetic conditions, well before the increase in magnetic activity on April 7, precludes any physical connection of the wind divergence with magnetic activity. The abatement in wind divergence that is observed on April 7, which is well correlated with the rise in magnetic activity, is interpreted by Portnyagin et al. (1997) as the signature of upwelling/convergence (Roble, 1992) due to Joule heating in the lower thermosphere (ca. 100-150 km).

The measurement of a net divergence of meridional winds in excess of $20 \mathrm{~m} \mathrm{~s}^{-1}$ implies extreme net vertical winds; a rough estimate for the vertical wind based on the zonal mean continuity equation is $\sim 0.7 \mathrm{~m} \mathrm{~s}^{-1}$. Therefore, our measurements imply extreme adiabatic heating/cooling rates and/or meridional heat fluxes over the South Pole mesopause region during this unusual event. Portnyagin et al. (1997) speculate that this event may be driven by intense wave 1 upward fluxes known to characterize the middle atmosphere near the autumnal equinox (Miles et al., 1994).

The left panel in Fig. 4 illustrates the monthly mean wind vectors, or, the zonally asymmetric component of the wind field, over the South pole during all the months of operation. The amplitudes of the vectors are of order 3-8 $\mathrm{m} \mathrm{s}^{-1}$ with errors less than $0.7 \mathrm{~m} \mathrm{~s}^{-1}$. A pattern of rotation of the wind vector about the pole is seen, extending from summer through equinox to winter months. The existence of a zonally-asymmetric mean wind over South Pole has been previously noted by Hernandez et al. (1992a), who suggest that such an observation would be consistent with a polar vortex displaced from the South geographic pole. Analyses of monthly mean geopotential height charts for the Southern Hemisphere lower thermosphere near $90 \mathrm{~km}$ (Koshelkov, 1984) indeed show that the polar vortex is displaced away from the pole. Moreover, it is observed that the anticyclonic vortex typical of summer months is destroyed in January/February, and in March is displaced with a cyclonic vortex. The right panel in Fig. 4 illustrates how the polar vortex might migrate and change direction to yield the the wind vectors in the panel to the left.

\section{Measurement Results-Traveling Wave Distur- bances in the Wind Field}

As illustrated in Portnyagin et al. (1998) and Palo et al. (1998), sliding spectrograms constructed for the first year of hourly measurements yield several classes of wave disturbances which represent regular features of the lower thermosphere over South Pole. The characteristics of these wave disturbances are now described.

\subsection{Diurnal migrating tide}

The amplitudes and phases of the 24-hour oscillation with 


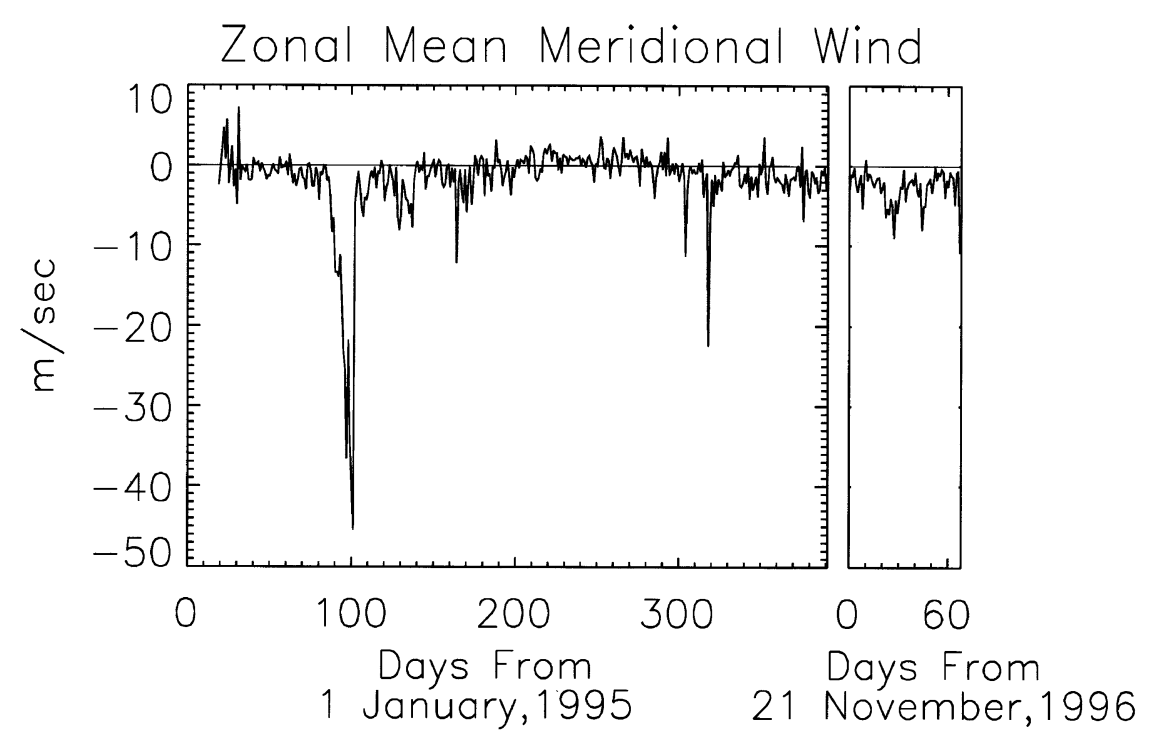

Fig. 2. Daily- and zonal-mean meridional wind observed over South Pole. Left: January 19, 1995-January 26, 1996. Right: November 21, 1996-January 27, 1997.

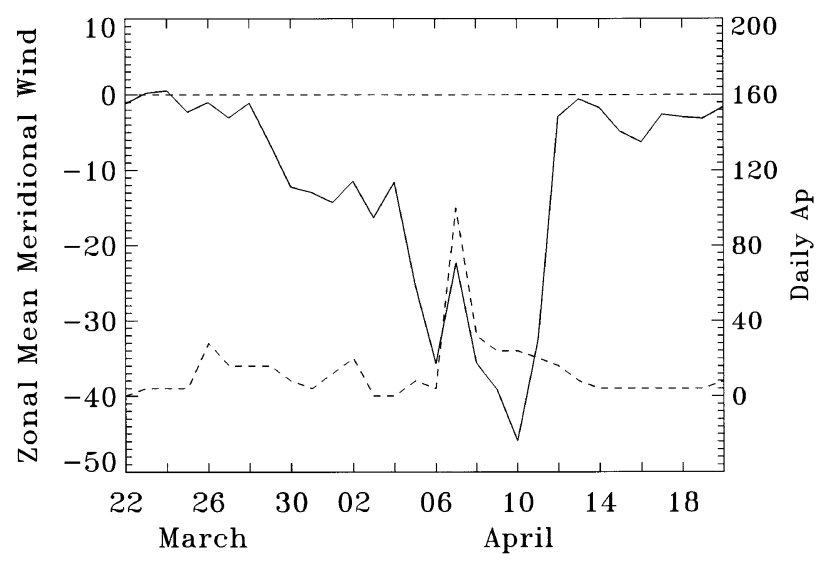

Fig. 3. As in Fig. 2, except focusing on March 22-April 20, 1995. The dashed line (right-hand scale) represents the daily global magnetic index, Ap. From Portnyagin et al. (1997).

zonal wavenumber $s=1$, averaged over sequential 10-day intervals, are illustrated in Fig. 5. This combination of wave period and zonal wavenumber yields a zonal phase speed equal to that of the apparent "migration" of the sun from the perspective of an earth-fixed observer; hence the term "migrating tide", which is synonymous with "sun-synchronous tide", although the latter phrase is seldom used. The amplitude and phase in Fig. 5 were obtained by least-squares fitting a function of the form

$$
A \cos \left[\omega\left(t-t_{\max }\right)+s \lambda\right]
$$

where $t=$ Universal Time, $t_{\max }=\mathrm{UT}$ of maximum at longitude $\lambda=0.0, \omega=$ wave frequency $=2 \pi / 24 \mathrm{~h}^{-1}$ and $s=$ zonal wavenumber $=1$, to the hourly wind data in all four directions simultaneously. According to this definition, phase $\left(t_{\max }\right)$ is defined as the UT of maximum at $\lambda=0$ (Greenwich). Alternatively, by a rearrangement of terms within the brackets, phase could be defined as the longitude of maximum at 0 UT (N.B. The migrating tidal structure is fixed in the sun-earth inertial frame (the earth rotates beneath it) and hence is independent of longitude; the dependence on longitude drops out of the above expression when UT is expressed in terms of LST (local solar time) and longitude: $\mathrm{UT}=\mathrm{LST}-12 \lambda / \pi$. This is of course consistent with the fact that UT and LST are the same at $\lambda=0$ ). The assumption that $s=1$ for the current data set is very good, as will be illustrated below for the semidurnal (12-hour) wind oscillation.

We note in Fig. 5 that the UT of maximum southward wind at $0^{\circ}$ longitude (which is the same as local time at $0^{\circ}$ longitude, and hence for a migrating tide, is the local time of maximum at all longitudes) ranges between about 1000 and 1500 hours. The diurnal wind amplitude is less than $3 \mathrm{~m} \mathrm{~s}^{-1}$ from mid-April through mid-August, and reaches maximum values of order $8-11 \mathrm{~m} \mathrm{~s}^{-1}$ during local summer. This behaviour is very similar to the climatology illustrated by Fraser et al. (1995) for the diurnal tide at Scott Base $\left(78^{\circ} \mathrm{S}, 167^{\circ} \mathrm{E}\right)$. Simulations from the Global Scale Wave Model (GSWM; Hagan et al., 1993; 1995) are also shown for comparison in Fig. 5. The phase of the oscillation is in excellent agreement with the GSWM, and the general amplitudes and salient behavior of the amplitude variation are also consistent between model and theory, except for the 2-month difference in the time of amplitude maximum near the beginning of the year (1 April for the model, and 1 February for the data). Thus, the origin and basic physics underlying the diurnal tide over South Pole appear to be reasonably understood. Note also the consistency in amplitudes and phases between the first and second years of operation of the meteor radar.

\subsection{Semidiurnal nonmigrating tide}

Our meteor radar data show that a large semidiurnal oscillation with zonal wavenumber $s=1$ occurs during local summer months. A typical example is provided in Fig. 6. 

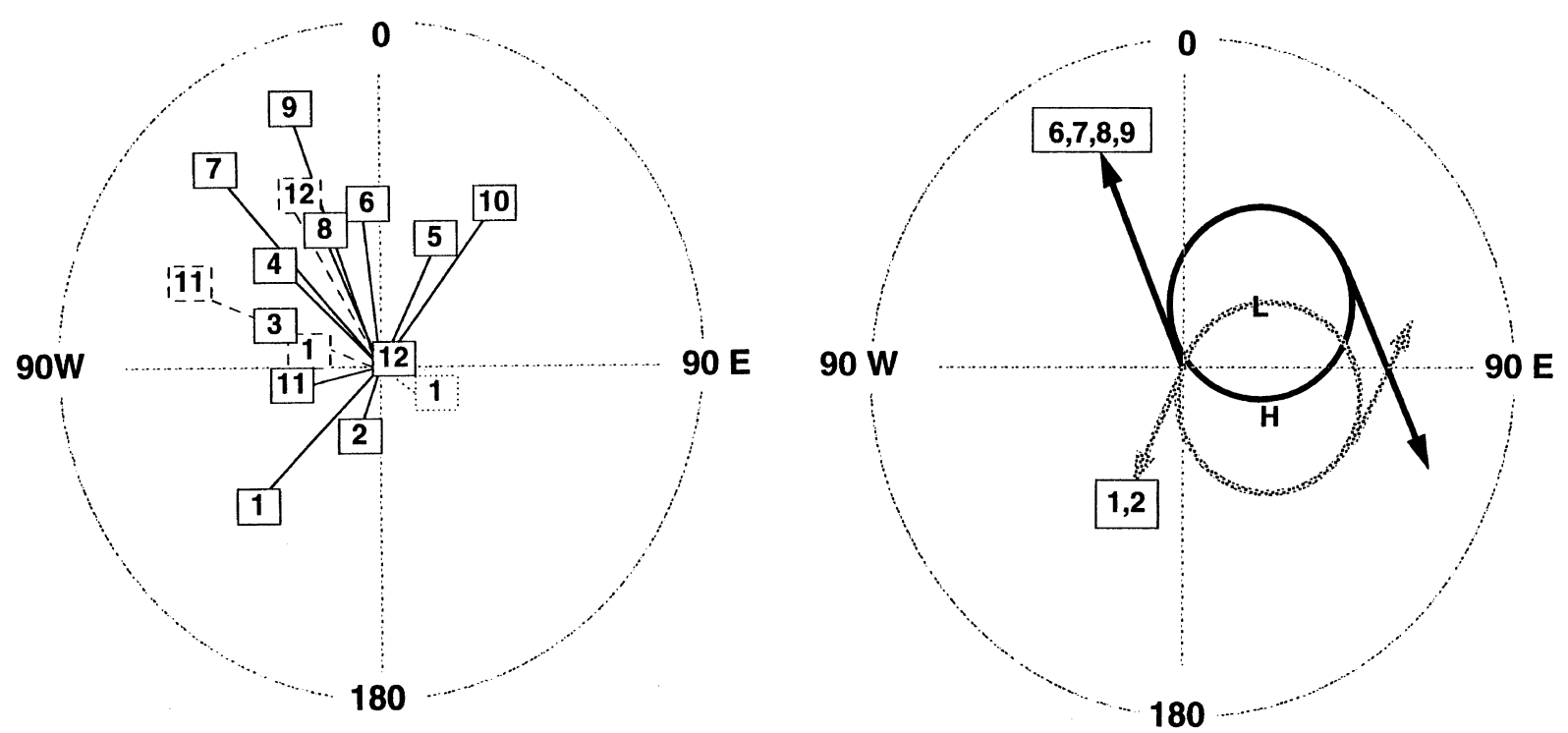

Fig. 4. Left: Monthly-mean zonally-asymmetric wind vectors over South Pole. The radius of the circle is $10 \mathrm{~m} \mathrm{~s}^{-1}$. Amplitude errors are less than $0.70 \mathrm{~m} \mathrm{~s}^{-1}$. Months are numbered 1-12. Solid lines: January 19-31, 1995 and February-December, 1995. Dotted lines: January 1-26, 1996. Dashed lines: November 21-30, 1996, December, 1996, and January 1-27, 1997. Right: Schematic illustrating how migration of the polar vortex with respect to the rotational pole can give rise to the differences in the vector of the zonally asymmetric wind between "summer" (months 1,2$)$ and "winter" (months $6,7,8,9)$ depicted in the left panel.
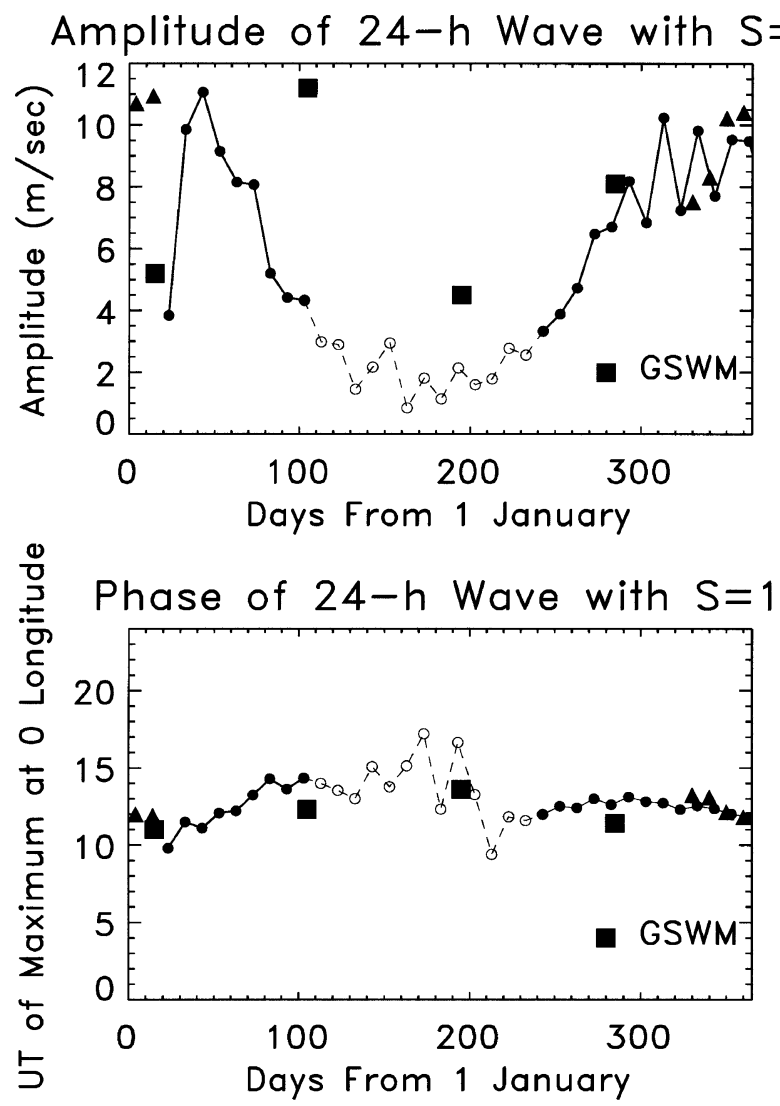

Fig. 5. Ten-day average amplitudes (top) and phases (bottom) of the diurnal (24-h) tide with zonal wavenumber $s=1$. Dots represent January 19, 1995-January 26, 1996, and triangles represent November 21, 1996-January 27,1997 . The open circles and dashed lines indicate periods when the amplitudes are less than $3 \mathrm{~m} \mathrm{~s}^{-1}$. The GSWM values for January, April, July and October are indicated by squares.
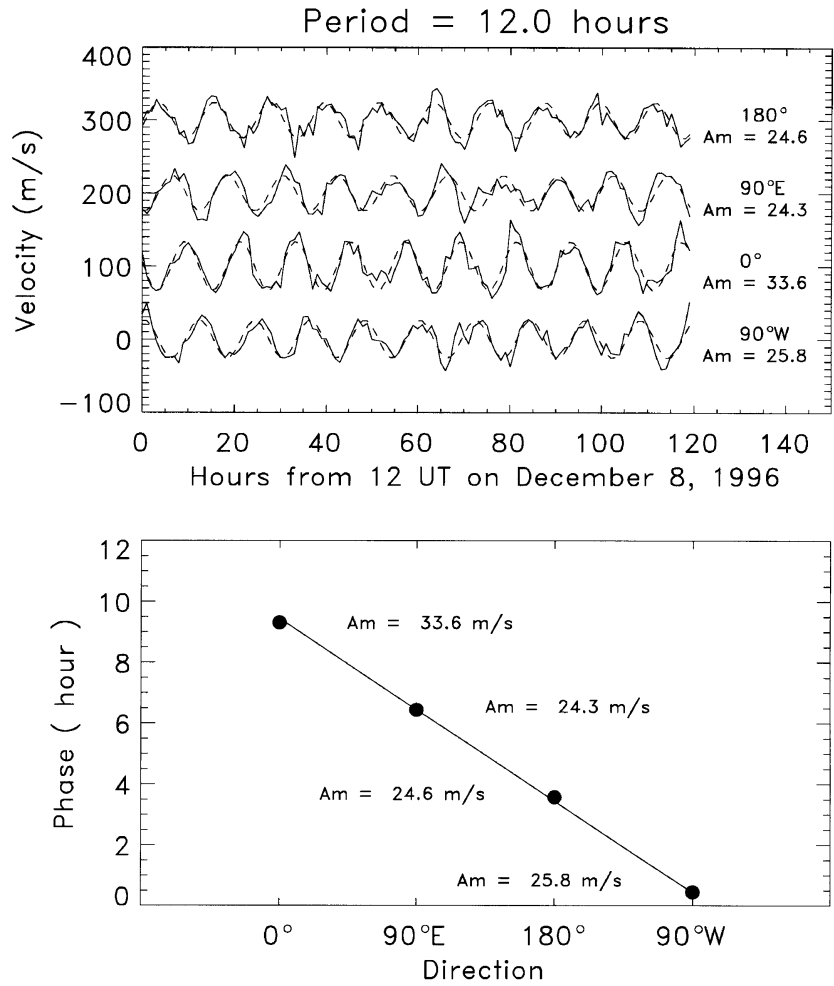

Fig. 6. Example of a 12-h oscillation during December 8-12, 1996. Top: Measured (solid line) hourly wind values in the four longitude directions, and corresponding fits with 12-h sinusoids (dashed lines). Bottom: Phases (UT of maximum) in the four directions. Solid line corresponds to westward propagation with $s=1$, and does not represent a fit to the data points. 
In this example the amplitude is of order $30 \mathrm{~m} \mathrm{~s}^{-1}$, is coherent in all four directions, with phase progression to the west consistent with a zonal wavenumber $s=1$. This stands in contrast to the migrating semidiurnal tide with $s=2$, which dominates at middle latitudes. Continuity and other theoretical constraints (Hernandez et al., 1992b, 1995; Forbes et al., 1999) preclude the existence of zonal wavenumbers two and higher at the rotational pole. Still, it is somewhat surprising that the amplitudes of the $s=1$ semidiurnal oscillation, several tens of $\mathrm{m} \mathrm{s}^{-1}$, are as large as the observed migrating oscillation at middle latitudes. We note that during winter the amplitudes are near the limits of detectability $\left(\sim 3 \mathrm{~m} \mathrm{~s}^{-1}\right)$. Wind oscillations with zonal wavenumber $s=1$ and periods between $11.6 \mathrm{~h}$ and $12.2 \mathrm{~h}$, and persisting over 10 days or more with amplitudes on the order of 6-7 $\mathrm{m} \mathrm{s}^{-1}$, have previously been observed over South Pole during August periods (Hernandez et al., 1993, 1997). As is now evident, these wintertime optical observations have occurred when the semidiurnal nonmigrating tide is weakest.

In the same format as Fig. 5, ten-day average amplitudes and phases of the semidurnal oscillation with $s=1$ over South Pole are presented in Fig. 7. These were obtained using the same fitting function (1) as for the diurnal tide, except assuming a frequency $\omega=2 \pi / 12 \mathrm{~h}^{-1}$. The wave amplitude maximizes during November, reaches a minimum in early January, and then a second maximum later in January. This general behavior is repeated for the second summer of
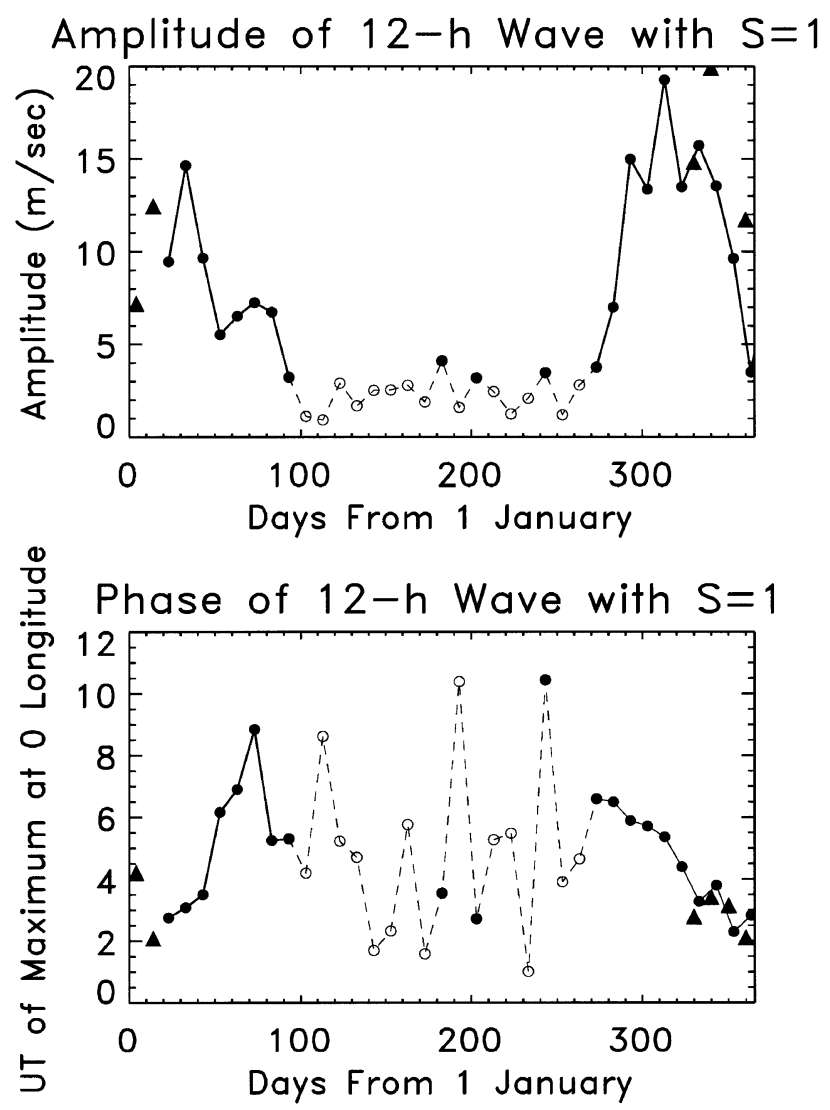

Fig. 7. Same as Fig. 5, except for semidiurnal (12-h) tide with $s=1$. GSWM simulations are not available for this wave component. observations, November, 1996-January, 1997, as indicated by the triangular points in Fig. 7. When the amplitude is larger than about $3 \mathrm{~m} \mathrm{~s}^{-1}$, the phase (UT of maximum at $0^{\circ}$ longitude) follows a smooth intraseasonal progression, from about $6.5 \mathrm{~h}$ in September to about $3.0 \mathrm{~h}$ in November, increasing to $6-8 \mathrm{~h}$ in January/February. This behavior is also repeated during the subsequent summer season.

As noted by Portnyagin et al. (1998), the annual and intraseasonal variations in amplitude and phase of the $s=1$ semidiurnal oscillation at South Pole are very similar to the climatological behavior observed by the MF radar at Scott Base (78 $\mathrm{S}$; Fraser et al., 1995), suggesting that the $s=1$ component of the semidiurnal oscillation may dominate as far as $12^{\circ}$ latitude from the pole. Conversely, the climatological amplitude and phase behaviors at stations near $67^{\circ} \mathrm{S}$ such as Mawson and Molodezhnaya are much different than at higher latitudes, suggesting that at these latitudes the $s=2$ component may be making a much stronger contribution. Resolution of these uncertainties requires coordinated simultaneous observations from the expanding network of Antarctic stations, so that longitude and latitude effects can be separated.

Portnyagin et al. (1998) also note significant day-to-day variability in the amplitude of the $12-\mathrm{h} s=1$ oscillation, at periods near 5, 7, 10 and 20 days, which is suggestive of planetary wave modulation of the 12 -h oscillation. However, such interpretations are severely hindered by our current lack of understanding concerning the origin of the 12-h oscillation with $s=1$. Three potential mechanisms have been suggested to date: (1) Nonlinear interaction between the migrating $(s=2)$ semidiurnal tide and a stationary $s=1$ feature (Forbes et al., 1995b). (Note that a polar vortex displaced from the rotational pole constitutes a stationary $s=1$ structure.); (2) An in-situ excitation source of unknown origin (Portnyagin et al., 1998); (3) Thermal excitation in the troposphere (Miyahara and Miyoshi, 1999). The reader is referred to Portnyagin et al. (1998) for more detailed discussions of each of these potential mechanisms.

\subsection{Long-period waves}

During winter months the observations often reveal the presence of oscillations with periods between about 1.5 and 10 days, all of which are propagating to the east with zonal wavenumber $s=1$. These waves apparently be long to the same class as the 2-3 day period oscillations measured by Hernandez et al. (1992b, 1995) and Fraser et al. (1993). The raw data during one such event is illustrated in Fig. 8, fit with a sinusoid of period equal to 2.3 days. The bottom panel of this figure demonstrates that this wave is eastwardpropagating. Palo et al. (1998) identify seven events which fall into this category. It is interesting to note that four of these wave events possess amplitudes which are about equal in all four directions, whereas the remaining cases indicate wave amplitudes in two directions, $180^{\circ}$ apart, to be significantly smaller than in the remaining two directions. One possible explanation for the latter behavior is that the observed wave is not propagating zonally around the geographic (rotational) pole, but rather is propagating around an off set dynamical pole.

The amplitude and phase characteristics of one additional type of long-period wave event observed over South Pole is illustrated in Fig. 9 (Palo et al., 1998). This wave had a period 

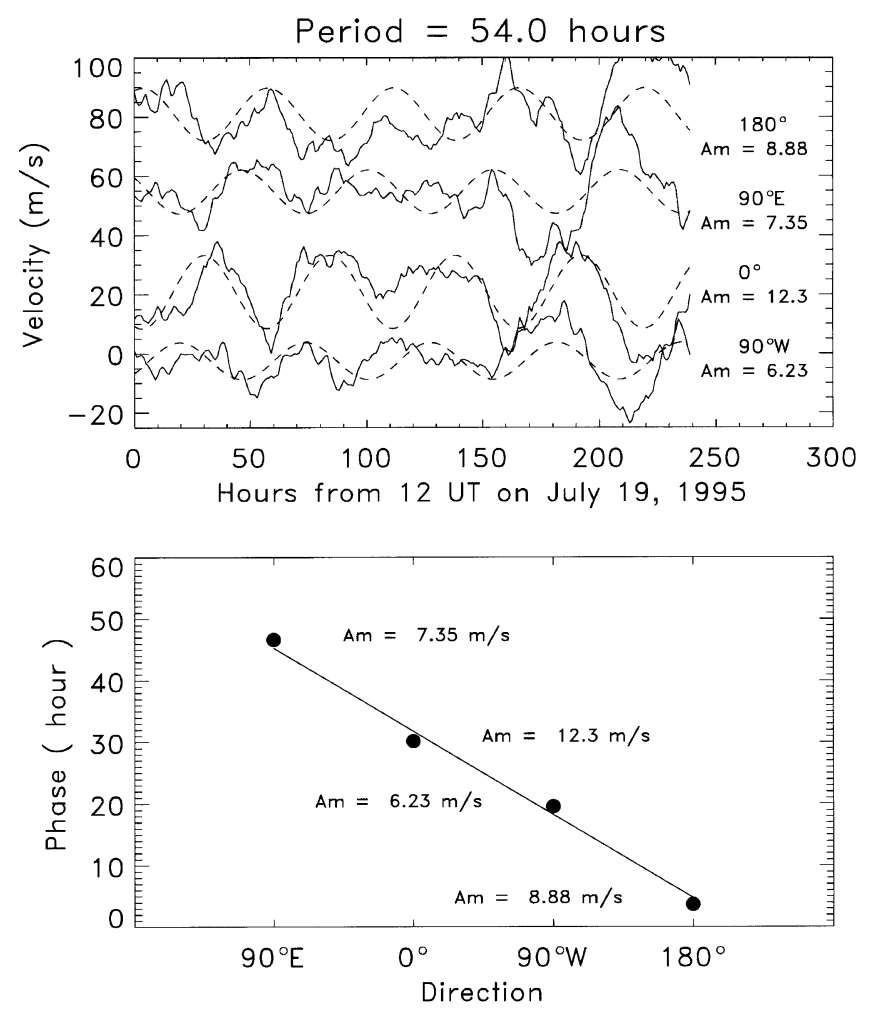

Fig. 8. Same as Fig. 6, except for eastward-propagating wave with period of 2.3 days during July 19-28, 1995. The winds displayed in the top panel represent five-hour running means of the hourly measurements.

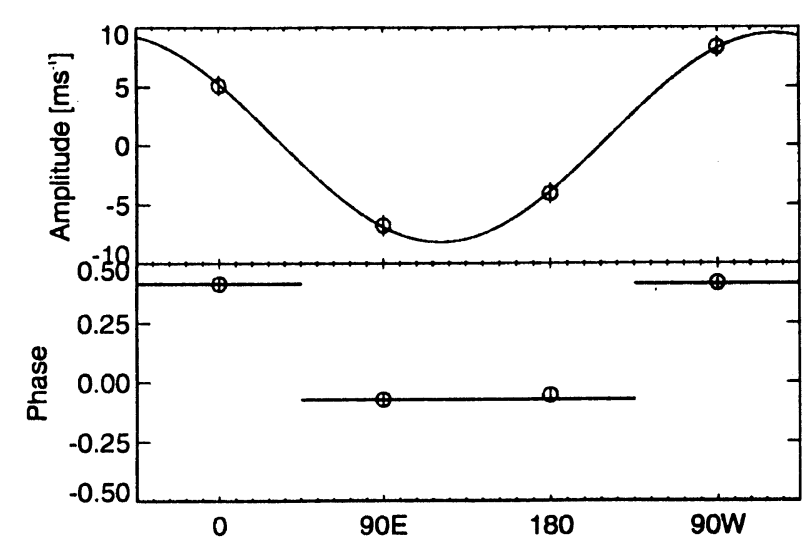

Fig. 9. Amplitude (top) and phase (bottom) of the 5.2-day eastward propagating oscillation during September, 1996, which is interpreted as a standing oscillation. (Palo et al., 1998).

of about 5.2 days, and lasted for about 25 days during September, 1996. The phase variation in Fig. 9 does not resemble that of an $s=1$ oscillation; rather, the phase is constant in pairs of longitudes where the two sets of longitude pairs are separated in phase by half a period. The amplitude variation is systematic and can be described by a sinusoidal variation. It appears that this is a mesospheric standing oscillation similar to the one observed by Hernandez et al. (1997). This type of wave can be viewed as a "sloshing back and forth" over the Pole, as opposed to zonal propagation around the
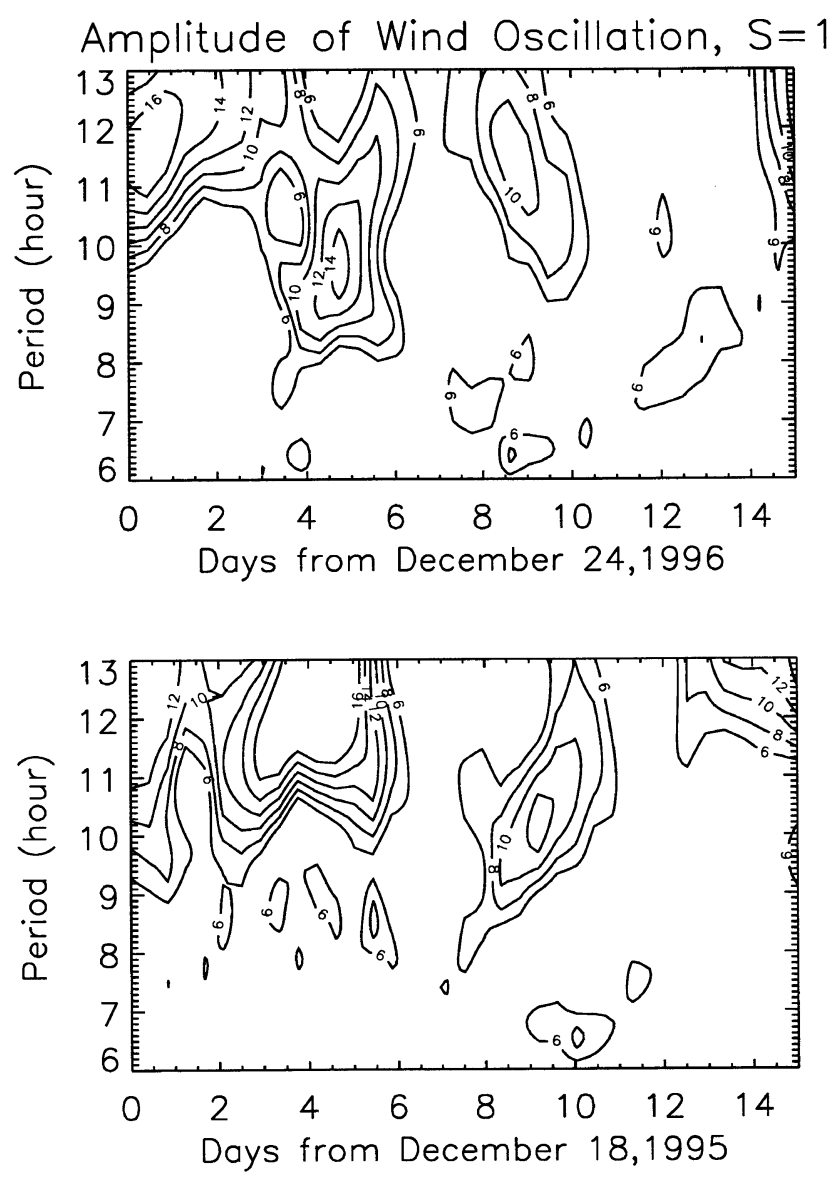

Fig. 10. Spectrograms illustrating intradiurnal oscillations propagating with $s=1$ during December, 1996 (top) and December, 1995 (bottom). An oscillation near 9.4-hour period occurs between December 28 and December 30, 1996. An oscillation near 10-hour period occurs between December 26 and December 28, 1995.

pole. The physical origin is unknown.

Periods of the oscillations discussed above are generally longer towards the equinoctial periods and shortest towards mid-winter. (Palo et al., 1998). As noted by Palo et al. (1998), this behavior is consistent with the interpretation that the waves are instability-driven. Analysis of the meridional gradient of quasi-geostrophic potential vorticity reveals a region in the high-latitude upper mesosphere which could support an instability and serve as a source for these waves, much in the same manner that the eastward-propagating 4day wave and its subharmonics are driven by a stratospheric instability (Manney et al., 1988; Manney and Randel, 1993). Given the limited data available, a stratospheric instability source for the eastward propagating waves considered here cannot be ruled out.

\subsection{Intradiurnal oscillations}

According to our meteor radar wind measurements, waves with periods between about 6 and 11.5 hours are common features of the meridional wind field over South Pole (Forbes et al., 1999; Portnyagin et al., 1999). The reader is referred to Forbes et al. (1999) for a more complete discussion of data presented here, methods of data analysis, statistical significance criteria, etc. Two typical examples are illustrated in the spectrograms comprising Fig. 10, which were obtained 

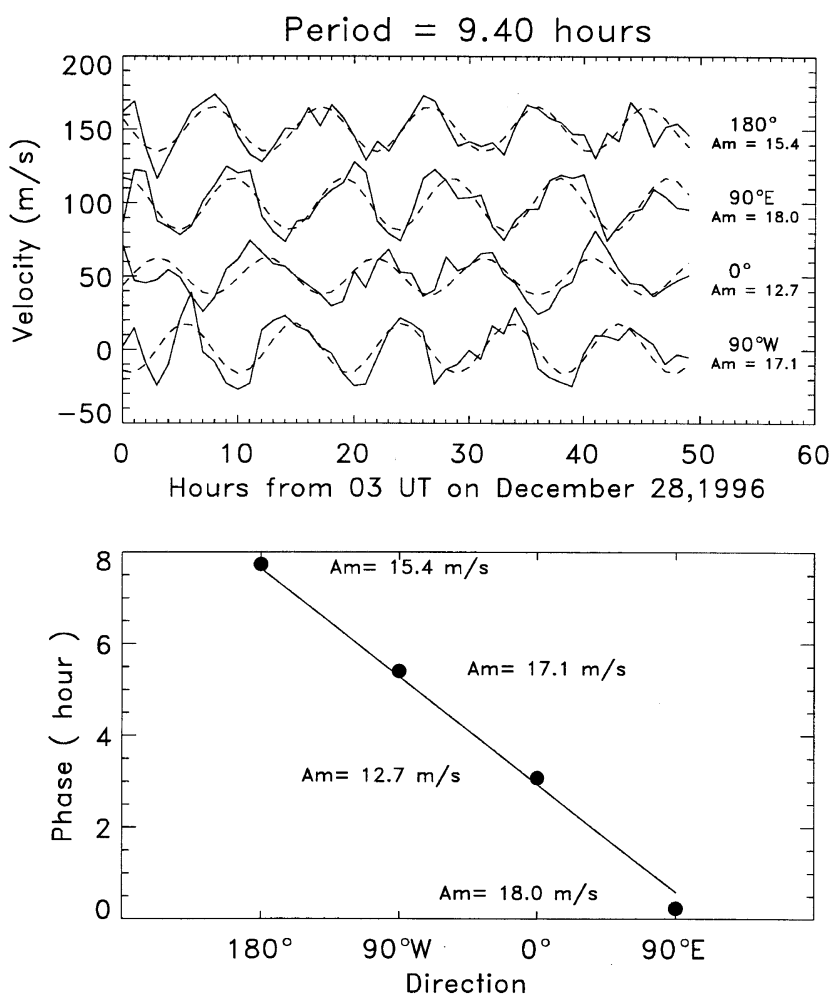

Fig. 11. Same as Fig. 6, except for the 9.4-hour oscillation depicted in the top panel of Fig. 10 .

by high-pass filtering the data with cutoff at 18 hour period, and constructing periodograms within a 50-hour sliding window with 40-hour overlap for successive spectra. There is a 9.4-hour oscillation occurring between December 28 and December 30, 1996, and a 10.1-hour oscillation occurring between December 26 and December 29, 1995. The larger oscillations in both of these figures correspond to the semidiurnal tide with $s=1$ discussed previously. Figure 11 illustrates the raw data corresponding to the former event, least-square fits of sinusoids to the data, and (in the lower panel) phases of the wind variations in the four directions, illustrating westward-propagating $s=1$ behavior.

The top panel of Fig. 12 illustrates the wave periods of all the intradiurnal oscillation events observed over South Pole for both measurement campaign periods of the meteor radar. Each point corresponds to a 50-hour fitting interval when the wave amplitude exceeds $7 \mathrm{~m} \mathrm{~s}^{-1}$, or twice the noise-level amplitude (see Forbes et al., 1999), and so some of the longer "events" may be represented by two or more points. The bottom panel depicts the disribution of wave amplitudes, which are generally limited to less than $15 \mathrm{~m} \mathrm{~s}^{-1}$. Considering that two full summer seasons are represented in Fig. 12, and that no such oscillations occur around the equinoxes, it is clear that the intradiurnal oscillations primarily represent a wintertime phenomenon.

The wave periods depicted in Fig. 12 range between 6 and 11.5 hours. However, the periods do not appear in discrete bands, as might be expected for tides. And, although tidal harmonics of 8 hours (terdiurnal) or 6 hours (quatradiurnal) appear within this range, the corresponding zonal wave num-

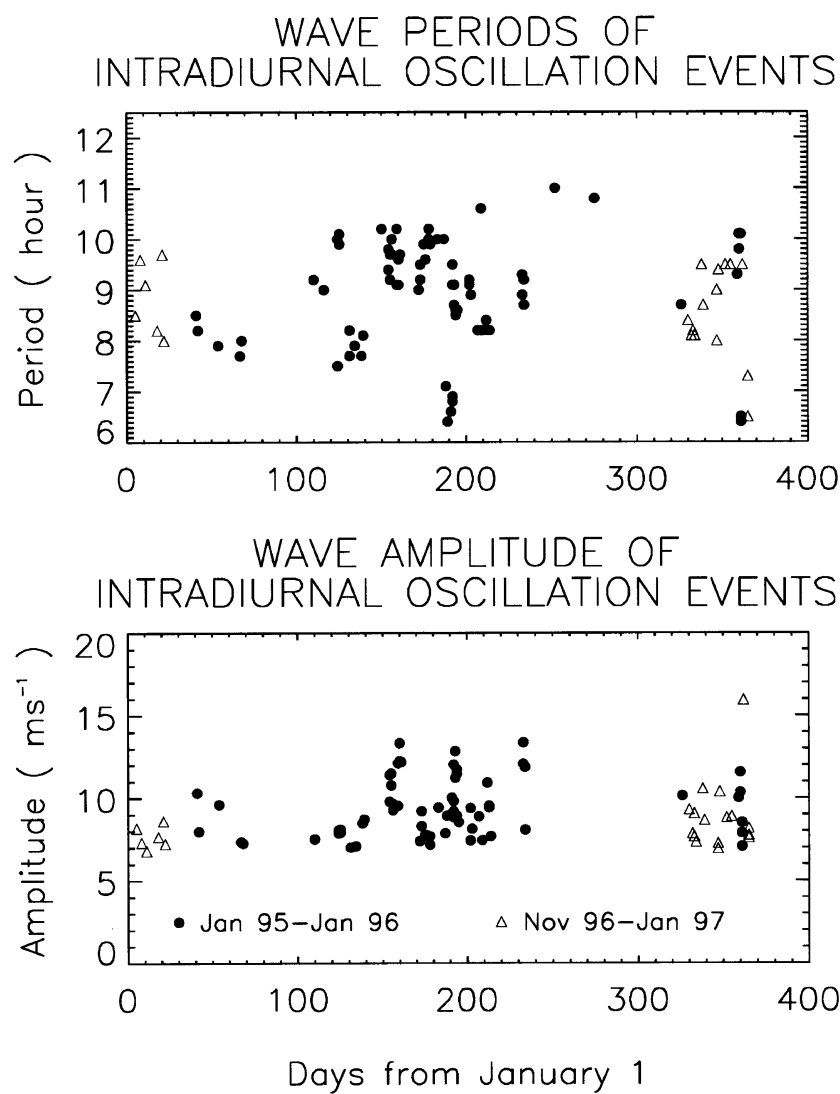

Fig. 12. Distributions of wave periods (top) and wave amplitudes (bottom) corresponding to westward-propagating $s=1$ oscillations with periods less than 12 hours. Solid circles correspond to January 19, 1995-January 26, 1996. Open triangles correspond to November 21, 1996-January 27, 1997.

bers of $s=3$ and $s=4$, respectively, are not expected to exist in the wind field at the pole, as discussed previously. However, there do exist normal modes of the "gravitational" type between 6 and 11.5 hours (Longuet-Higgins, 1968), and which have recently been shown to represent the preferred response to broadband forcing in the thermosphere (Meyer and Forbes, 1997). These short-period normal modes are sometimes referred to as "Lamb" waves (Lindzen and Blake, 1972). Waves with period near 10 hours have been observed previously over South Pole by Hernandez et al. (1992b, 1993, 1995). For the August 1-25, 1991, experiment, these authors utilized the Scott Base $\left(78^{\circ} \mathrm{S}\right)$ radar observation of a $\sim 100$ $\mathrm{km}$ vertical wavelength for this oscillation, close to that expected for a normal mode in a realistic atmosphere at these heights, to support interpretation as a normal mode.

For $s=1$ and an equivalent depth of $10 \mathrm{~km}$, the normal modes referred to above include the second symmetric mode at a period of $8.6 \mathrm{~h}$, and the first and second asymmetric modes with periods of $10.4 \mathrm{~h}$ and $7.2 \mathrm{~h}$, respectively. Moreover, normal mode periods can vary depending on background mean wind conditions due to Doppler-shifting effects (see Forbes (1995) for a simple analytic explanation), which are especially pronounced at high latitudes. Therefore the non-discrete distribution of wave periods in Fig. 12 is not necessarily inconsistent with a normal mode interpretation.

It is possible to utilize the GSWM to simulate the global 


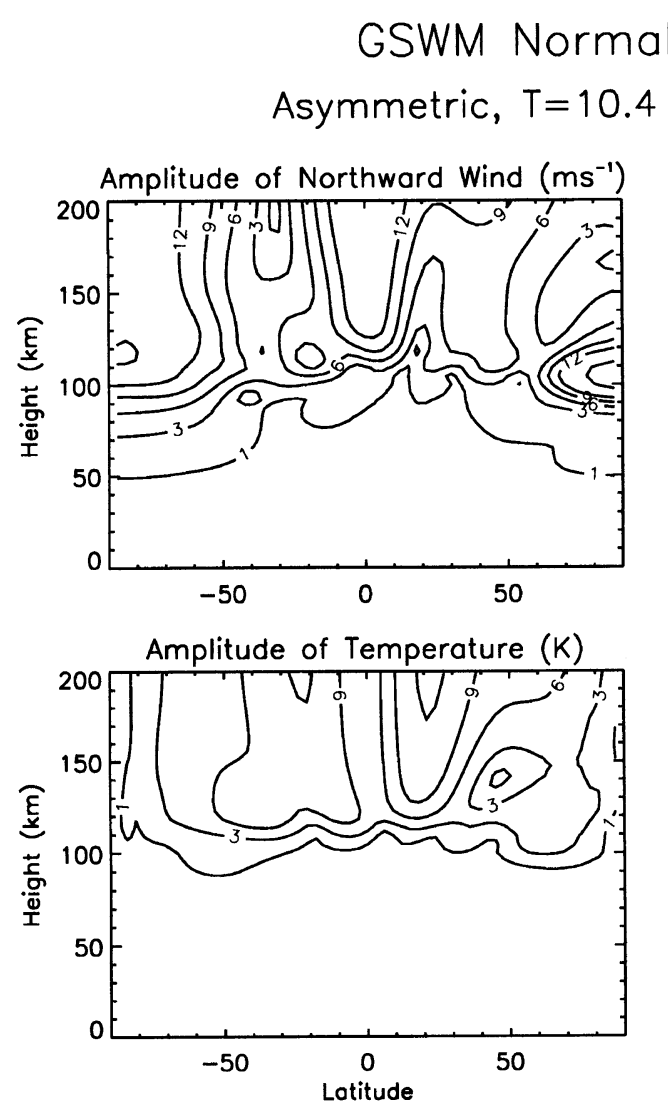

\section{Mode Simulation}

hours, $S=1$, Month= July
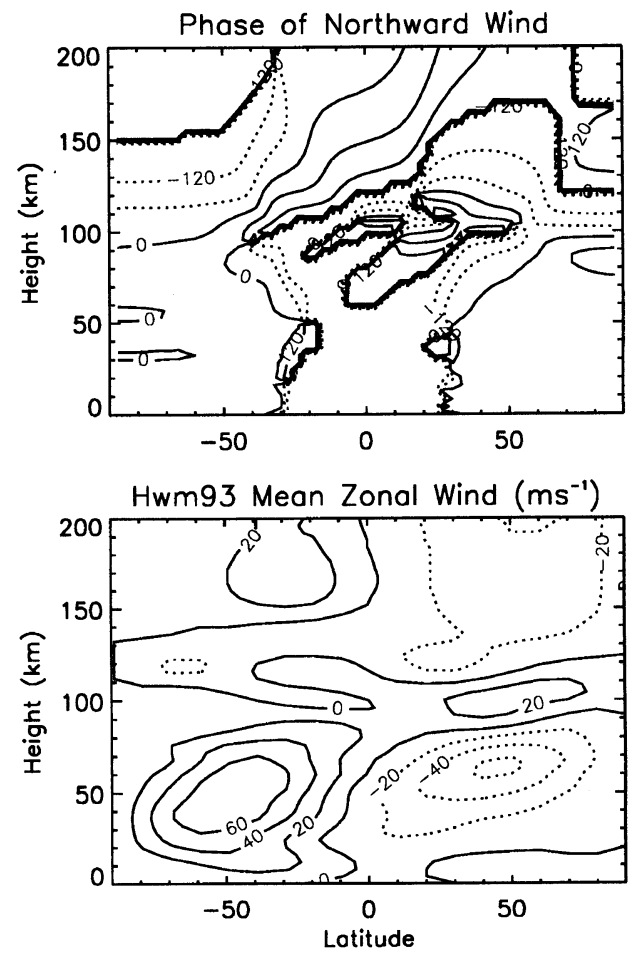

Fig. 13. GSWM simulation of the 10.4-hour gravitational normal mode.

structure of normal modes. This has been done previously for the 2-day wave (Hagan et al., 1993) and the 16-day wave (Forbes et al., 1995a). It is known from these works and further studies by the authors that the upper-level response is insensitive to the method of forcing (i.e., heating profile or vertical motion at the surface) and to the horizontal shape of forcing. For the present simulation of the 10.4-hour wave, illustrated in Fig. 13, the normal mode shape was assumed for the latitude dependence of the tropospheric thermal forcing which was assumed to have a Gaussian shape in the vertical with a peak near $6.5 \mathrm{~km}$. The amplitude of forcing was adjusted to yield a $10 \mathrm{~m} \mathrm{~s}^{-1}$ amplitude for the meridional wind at $95 \mathrm{~km}$ over South Pole, i.e., close to that observed. July conditions were assumed, and the MSISE-90 model (Hedin, 1991) and the HWM-93 model (Hedin et al., 1996) were adopted to specify the background atmospheric state.

There are several interesting features worth noting in Fig. 13. The phase behavior of the northward wind is evanescent up to about $80-90 \mathrm{~km}$, but then reflects propagating characteristics (phase progression) at higher altitudes. This is consistent with the shift to a strong positive thermal gradient in the lower thermosphere (Forbes, 1995). With a $10 \mathrm{~m} \mathrm{~s}^{-1}$ northward wind amplitude over South Pole, the maximum amplitude on a global scale (about $24 \mathrm{~m} \mathrm{~s}^{-1}$ ) is predicted to occur in the northern polar region $\left(70^{\circ}-90^{\circ}\right.$ latitude) between about 95 and $115 \mathrm{~km}$. Thus, this oscillation is potentially observable by the EISCAT radar system. Maximum temperatures associate with this mode occur near $18^{\circ}$ latitude and between 130 and $150 \mathrm{~km}$, and are thus potentially observable by the Arecibo incoherent scatter radar. However, it is likely that significant semidiurnal oscillations normally exist at both these locations. Hence a 10.4-hour oscillation, if it has ever existed during an observational campaign, could have been embedded in the data and gone unnoticed.

\subsection{Comparison with optical measurements}

A comparison between winds near $88 \mathrm{~km}$ altitude measured by a colocated high resolution $\mathrm{OH}$ spectrometer, and our meteor radar winds near $95 \mathrm{~km}$ was conducted during 12 consecutive days in the winter of 1995 (Hernandez et al., 1996). This comparison indicated a correlation coefficient between the two sets of measurements of $r=0.82$ at the $99.99 \%$ significance level. An analysis was also conducted for three significant wave periods ( 2.45 days, 3.2 days and 10.9 hours) that were identified during the comparison interval. Note that the first two waves are of the long-period eastward-propagating type, and the third is interpreted as a gravitational normal mode, as discussed above. The amplitudes of the 2.45 and 3.2-day oscillations measured by the $\mathrm{OH}$ spectrometer are $11.3 \pm 10.7 \mathrm{~m} \mathrm{~s}^{-1}$ and $11.6 \pm 1.3 \mathrm{~m} \mathrm{~s}^{-1}$, and are $9.0 \pm 1.5 \mathrm{~m} \mathrm{~s}^{-1}$ and $7.9 \pm 0.6 \mathrm{~m} \mathrm{~s}^{-1}$ for the meteor radar. These differences suggest a slight decrease in wave amplitudes with height from 88 to $95 \mathrm{~km}$. A comparison of the phases for all three oscillations and the amplitudes for the 10.9-hour oscillation are provided in Fig. 14. The amplitudes of the 10.9-hour oscillation estimated by the two techniques are almost indistinguishable. There is a 

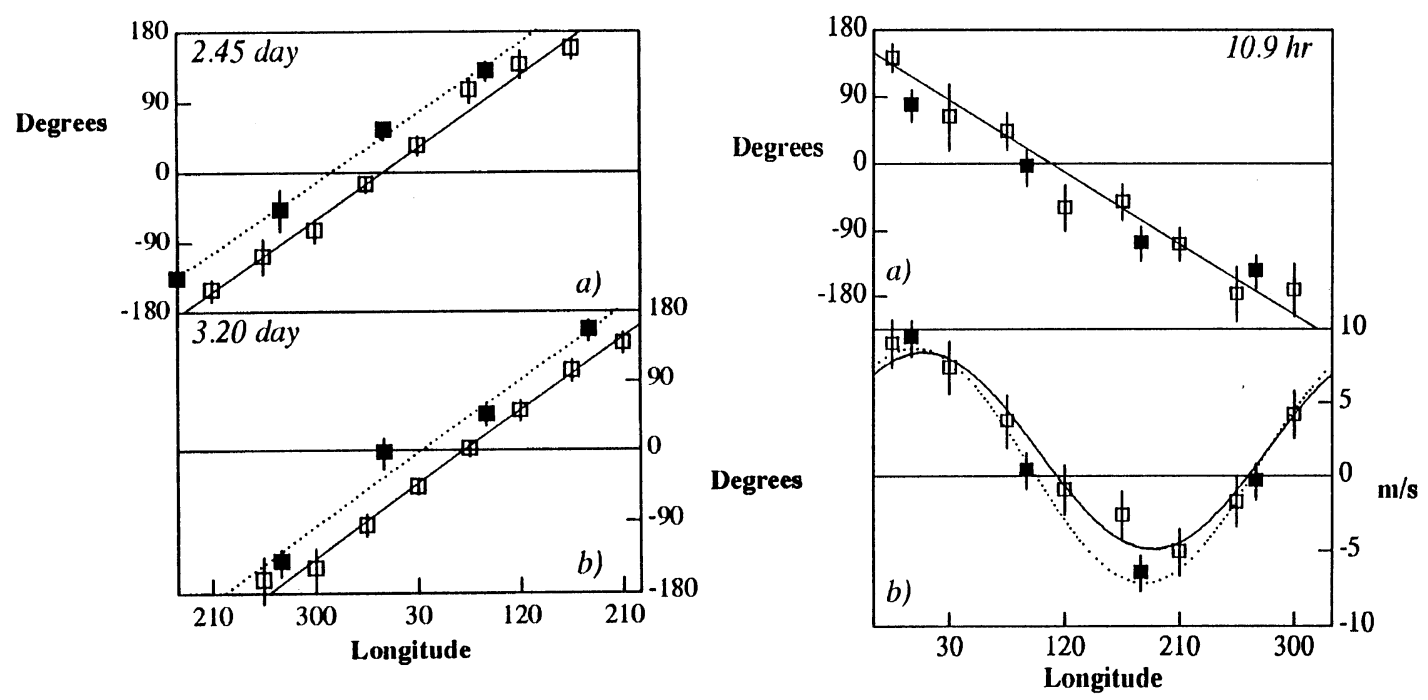

Fig. 14. Comparison between optical (solid symbols) and meteor radar (open symbols) measurements of winds near $88 \mathrm{~km}$ and $95 \mathrm{~km}$, respectively, over South Pole during June 19-30, 1995. (Hernandez et al., 1996).

slight shift in the phases observed by the two techniques for the eastward-propagating waves, which suggests downward phase progression with a vertical wavelength of about $65-70$ $\mathrm{km}$. Measured phases for the 10.9-hour wave are indistinguishable within the scatter of the measurements, suggesting a somewhat larger vertical wavelength than $70 \mathrm{~km}$.

\section{Conclusions}

The occurrence of 2-3 day eastward propagating and 10hour and 12-hour westward propagating wind oscillations with zonal wavenumber $s=1$ have previously been measured during winter clear-sky periods over the South Pole (Hernandez et al., 1992a, 1992b, 1993, 1995, 1996, 1997; Fraser et al., 1993). These and a number of other interesting dynamical phenomena occurring in the lower thermosphere over South Pole have been revealed by our meteor radar wind measurements. These include:

1) Occasional periods of intense divergence of zonally symmetric mean meridional winds;

2) Regular intra-annual variations in the zonally asymmetric component of the monthly mean wind, perhaps associated with seasonal migration of the polar vortex;

3) The 24-h migrating $(s=1)$ diurnal tide during nonwinter months, which appears to be reasonably well understood in terms of theory and modeling;

4) The nonmigrating westward-propagating semidiurnal oscillation with zonal wavenumber $s=1$ with largest amplitudes during non-winter months, with regular intraseasonal behavior, day-to-day variability at planetary wave periods, and repeatable characteristics from year to year;

5) Eastward-propagating waves with periods between 1.5 and 10 days, which may originate from instabilities in the mesosphere and/or stratosphere;
6) Westward-propagating intradiurnal oscillations with periods between 6 and 11.5 hours and zonal wavenumber $s=1$, which are tentatively identified as gravitational normal modes, or "Lamb" waves.

Except for the migrating diurnal tide, no defnitive explanations exist for any of the above phenomena. Further modeling work is needed, as well as correlative analyses with data from other Antarctic stations. A major question to emerge from the present study is:

To what extent do these phenomena exist in the Arctic lower thermosphere?

Acknowledgments. This work was partly supported by the National Science Foundation (Grants OPP-9320879 and ATM9728993) and by Russian Fund for Fundamental Researches (Grant N 96-05-64301).

\section{References}

Collins, R. L., D. C. Senft, and C. S. Gardner, Observations of a 12-hour wave in the mesopause region at the South Pole, Geophys. Res. Lett., 19, 57-60, 1992.

Forbes, J. M., Tidal and Planetary Waves, in The Upper Mesosphere and Lower Thermosphere: A Review of Experiment and Theory, Geophysical Monograph, 87, AGU, Washington D.C., 1995.

Forbes, J. M., M. E. Hagan, S. Miyahara, F. Vial, A. H. Manson, C. E. Meek, and Yu. I. Portnyagin, Quasi 16-day oscillation in the mesosphere and lower thermosphere, J. Geophys. Res., 100, 9149-9163, 1995a.

Forbes, J. M., N. A. Makarov, and Yu. I. Portnyagin, First results from the meteor radar at south pole: a large 12-hour oscillation with zonal wavenumber one, Geophys. Res. Lett., 22(23), 3247-3250, 1995b.

Forbes, J. M., S. E. Palo, X. Zhang, Yu. I. Portnyagin, N. A. Makarov, and E. G. Merzlyakov, Lamb Waves in the Lower Thermosphere: Observational Evidence and Global Consequences, J. Geophys. Res., 104(A8), 17,10717,116, 1999.

Fraser, G. J., G. Hernandez, and R. W. Smith, Eastward-moving 2-4 day waves in the winter Antarctic, Geophys. Res. Lett., 20(15), 1547-1550, 1993.

Fraser, G. J., Yu. I. Portnyagin, J. M. Forbes, R. A. Vincent, I. A. Lysenko, and N. A. Makarov, Diurnal tide in the Antarctic and Arctic mesosphere/lower thermosphere regions, J. Atmos. Terr. Phys., 57(4), 383-393, 1995. 
Hagan, M. E., J. M. Forbes, and F. Vial, Numerical investigation of the propagation of the quasi-two-day wave into the lower thermosphere, $J$. Geophys. Res., 98(D12), 23,193-23,205, 1993.

Hagan, M. E., J. M. Forbes, and F. Vial, On modeling the migrating solar tides, Geophys. Res. Lett., 22, 893-896, 1995.

Hedin, A. E., Extension of the MSIS thermosphere model into the middle and lower atmosphere, J. Geophys. Res., 96, 1159-1172, 1991.

Hedin, A. E., E. L. Fleming, A. H. Manson, F. J. Schmidlin, S. K. Avery, R. R. Clark, S. J. Franke, G. J. Fraser, T. Tsuda, F. Vial, and R. A. Vincent, Empirical wind model for the upper, middle and lower atmosphere, $J$. Atmos. Terr. Phys., 58(13), 1421-1448, 1996.

Hernandez, G., R. W. Smith, and J. Conner, Neutral wind and temperature in the upper atmosphere above South Pole, Antarctica, Geophys. Res. Lett., 19(1), 53-56, 1992a.

Hernandez, G., R. W. Smith, G. J. Fraser, and W. L. Jones, Large-scale waves in the upper mesosphere at Antarctic high latitudes, Geophys. Res. Lett., 19(13), 1347-1350, 1992b.

Hernandez, G., G. J. Fraser, and R. W. Smith, Mesospheric 12-hour oscillations near South Pole, Antarctica, Geophys. Res. Lett., 20(17), 17871790, 1993.

Hernandez, G., R. W. Smith, and G. J. Fraser, Antarctic high-latitude mesospheric dynamics, Adv. Space Res., 16(5), 71-80, 1995.

Hernandez, G., J. M. Forbes, R. W. Smith, Yu. I. Portnyagin, J. F. Booth, and N. A. Makarov, Simultaneous mesospheric wind measurements near South Pole by optical and meteor radar measurements, Geophys. Res. Lett., 23(10), 1079-1082, 1996.

Hernandez, G., R. W. Smith, J. M. Kelley, G. J. Fraser, and K. C. Clark, Mesospheric standing waves near South Pole, Geophys. Res. Lett., 24(16), 1987-1990, 1997.

Koshelkov, Yu. P., Climatology of the middle atmosphere of the Southern Hemisphere, J. Atmos. Terr. Phys., 46, 781-798, 1984.

Lindzen, R. S. and D. Blake, Lamb waves in the presence of realistic distributions of temperature and dissipation, J. Geophys. Res., 77(12), 21662176, 1972

Longuet-Higgins, M. S., The eigenfunctions of Laplace's tidal equation over asphere, Phil. Trans. R. Met. Soc. London, A262, 511-607, 1968.
Manney, G. L. and W. J. Randel, Instability at the winter stratopause: A mechanism for the 4-day wave, J. Atmos. Sci., 50, 3928-3938, 1993.

Manney, G. L., T. R. Nathan, and J. L. Standford, Barotropic instability of realistic stratospheric jets, J. Atmos. Sci., 45, 2545-2555, 1988.

Meyer, C. K. and J. M. Forbes, Natural oscillations of the ionospherethermosphere-mesosphere (ITM) system, J. Atmos. Sol.-Terr. Phys., 59, 2185-2302, 1997.

Miles, T., W. L. Grose, E. E. Remsberg, and G. Lingenfelser, Evolution of the Southern Hemisphere subpolar middle atmosphere during summer and autumn, J. Atmos. Sci., 51, 677-693, 1994.

Miyahara, S. and Y. Miyoshi, Migrating and nonmigrating atmospheric tides simulated by a middle atmosphere general circulation model, Adv. Space Res., 1999 (in press).

Palo, S. E., Yu. I. Portnyagin, J. M. Forbes, N. A. Makarov, and E. G. Merzlyakov, Transient eastward-propagating long-period waves observed over South Pole, Ann. Geophys., 16, 1486-1500, 1998.

Portnyagin, Yu. I., J. M. Forbes, and N. A. Makarov, Unusual characteristics of lower thermosphere prevailing winds at South Pole, Geophys. Res. Lett., 24(1), 81-84, 1997.

Portnyagin, Yu. I., J. M. Forbes, N. A. Makarov, E. G. Merzlyakov, and S. E. Palo, The summertime 12-hour wind oscillation with zonal wavenumber $s=1$ in the lower thermosphere over the South Pole, Ann. Geophys., 16, 828-837, 1998.

Portnyagin, Yu. I., J. M. Forbes, E. G. Merzlyakov, N. A. Makarov, and S. E. Palo, Intradiurnal wind variations observed in the lower thermosphere over South Pole, Ann. Geophys., 1999 (in press).

Roble, R. G., The polar lower thermosphere, Planet. Space Sci., 40, 271 297, 1992.

Sivjee, G. G. and R. L. Walterscheid, Six-hour zonally symmetric tidal oscillations of the winter mesopause over the South Pole Station, Planet. Space Sci., 42, 447-453, 1994.

J. M. Forbes (e-mail: forbes@zeke.colorado.edu), Yu. I. Portnyagin, N. A. Makarov, S. E. Palo, E. G. Merzlyakov, and X. Zhang 